\title{
Measurement of optical constants of steel with application to gauge block calibration
}

\author{
N. Mahmoud, N. Farid", N. Nagib \\ Length and Engineering Precision, National Institute for standards (NIS), Tersa St. Haram, Code:12211, \\ P.O.Box: 136 Giza, Egypt. \\ ${ }^{*}$ E) E-mail: niveen_farid@hotmail.com
}

Received: $19 / 05 / 2015 \quad$ Accepted: 01/07/2016

DOI: 10.7149/OPA.49.3.48542

\begin{abstract}
:
A simple technique for measuring the phase change on reflection with application to gauge block calibration is presented. The proposed technique measures the optical constants of the steel gauge block and the auxiliary platen which is made of fused silica using polarized light. The presented method considers the principal angle of incidence method and avoids the deviation of the quarterwave plate from exact $\lambda / 4$ value. The phase change angle could be determined with high accuracy $\left( \pm 2^{\circ}\right)$ and the uncertainty contribution of the phase change correction in the gauge block calibration could be reduced to $( \pm 2 \mathrm{~nm})$. The resulted phase change correction value was compared to the value obtained by interferometry using the stack method. The presented method is proposed to be convenient with the contactless measurement of gauge block length using the double ended interferometer.
\end{abstract}

Key words: Phase change correction, optical constants, polarized light, gauge block

\section{REFERENCES AND LINKS}

[1] P. Phuaknoi, S. Phiengbangyang, J. Buajarern and A. Tonmueanwai, "Determination model of phasechange correction for high precision gauge block calibration," The Journal of Industrial Technology, 8, 61-68(2012).

[2] T. Doiron, J. Beers, The gauge block handbook, http://emtoolbox.nist.gov/Publications/NISTMonograph180.pdf

[3] E. G. Thwaite, "Phase correction in the interferometric measurement of end standards," Metrologia, 14, 53-62(1978). http://dx.doi.org/10.1088/0026-1394/14/2/002

[4] K V Rajesh, R. J. Hocken, A study of gage block wringing, http://www.aspe.net/publications/Annual 2005/posters/3metro/6surf/1831.pdf.

[5] J. E. Decker, G. Bönsch, "On the influence of gauge blocks Roughness characteristic and surface treatment on the length calibration of optical interferometry," Proc. SPIE 5879, Recent Developments in Traceable Dimensional Measurements III, 587907 (2005); http://dx.doi.org/10.1117/12.614402

[6] G J Siddall and P C T Willey, "Flat surface wringing and contact error variability," J. Phys. D: Appl. Phys. 3, 8-28(1970) http://dx.doi.org/10.1088/0022-3727/3/1/303

[7] K. M. Medicus, A. Fricke, J. Brodziak, A. Davies, "The Effect of Phase Change on Reflection on Optical Measurements", Appl. Optics, 46, 2027-2035(2007). http://dx.doi.org/10.1364/A0.46.002027

[8] T. McWaid, T. Vorburger, J.F. Song, D. Chandler-Horowitz. "The effects of thin films on interferometric step height measurements," SPIE Interferometry: Surface Characterization and Testing, 1776, 213(1992).

http://dx.doi.org/10.1117/12.139238 
[9] J. M. Bennett, "Precise method for measuring the absolute phase change on reflection", J. Opt. Soc. Am, 54, 612-622(1964). http://dx.doi.org/10.1364/JOSA.54.000612

[10] S. Ishikawa, G. Bönsch, H. Böhme "Phase-shifting interferometry with a coupled interferometer: Application to optical roughness of gauge blocks", Optik, 91, 103-108(1992).

[11] J. B. Saunders, A high-sensitivity interferometer for measurement of phase-shift and other applications, NBS Circular, 581, 51-59(1957).

[12] G. Bönsch, Interferometric calibration of an integrating sphere for the determination of the roughness correction of gauge blocks, Proc. SPIE 3477, Recent Developments in Optical Gauge Block Metrology, 152-160 (1998). http://dx.doi.org/10.1117/12.323104

[13] A. Abdelaty, A. Walkov, P. Franke and R. Schödel, "Challenges on double ended gauge block interferometry unveiled by the study of a prototype at PTB,"Metrologia, 49, 307-314(2012) http://dx.doi.org/10.1088/0026-1394/49/3/307

[14] H. Hussein, N. Farid, and O. Terra, "Absolute gauge block calibration using ultra-precise optical frequency synthesizer locked to a femtosecond comb," Appl. Opt., 54, 622-626 (2015). http://dx.doi.org/10.1364/A0.54.000622

[15] D. H.Goldstein. Polarized light. 3rd ed. CRC Press, 481-9(2011).

[16] P. Frederick and R.C. Plumb, "Determining optical constants of metals by reflection of elliptically polarized light,” J. Opt. Soc. Am., 54, 1063-1064 (1964). http://dx.doi.org/10.1364/JOSA.54.001063

[17] N. N. Nagib, M. S. Bahrawi, H. Osman, N. A. Mahmoud, M. H. Osman and A. W. Abdallah, "A precise method for determining the principal angle of incidence and the optical constants of metals," Meas. Sci. Technol. 27, 015009 (2016). http://dx.doi.org/10.1088/0957-0233/27/1/015009

[18] I. H. Malitson, "Interspecimen comparison of the refractive index of fused silica," J. Opt. Soc. Am., 55, 1205-1209(1965). http://dx.doi.org/10.1364/JOSA.55.001205

[19] L. Ward, The Optical Constants of Bulk Materials and Films, Adam Hilger, Bristol, (1988).

[20] E. Palik, Handbook of Optical Constants of Solids, Academic, San Deigo, CA, )1985).

[21] D.E. Gray, Ed, American Institute of Physics Handbook. McGraw-Hill, New York, 3rd Edition, (1982).

[22] D.R. Lide, Ed., CRC Handbook of Chemistry and Physics. CRC Press, Boca Raton, 84th Edition, (2003).

[23] ISO: Guide to the expression of uncertainty in Measurements, International Standardizations Organization Publications (1995).

\section{Introduction}

Phase change on reflection is an important correction in the optical measurements of dimensions such as thickness, length, curvature. Such measurements depend on the material properties and the optical constants of the object of interest. Since gauge blocks (GBs) are necessary in manufacturing and treatability maintaining in dimensional metrology, they must be calibrated to high accuracy level using interferometry. In the calibration of a gauge block central length using interferometry, phase change parameter exists when the materials of the GB and the platen used in the wringing of the GB are different. In this case, the beams reflected from the surfaces of the GB and the platen are shifted in phase, and this shift induces an additional error to the measured central length. The correction depends on the characteristics of both GB and the auxiliary platen to which the GB is wrung. Such characteristics are the optical constants and the surface roughness of both pieces, Figure 1 [1].

The phase change error can be measured using the stack method [2] where a set of small GBs of same material are wrung together on the surface of the platen to form the stack. This increases the difficulty in obtaining good wringing. The error induced by the wringing must be concerned as it influences the uncertainty in the calibration of the GB block by $20 \mathrm{~nm}$ [3-5]. Moreover, the repeated wringing of the GB to the platen reduces the quality of its working faces and affects its length [6]. In [7], the phase change value was measured based on the incident angle, material properties and penetration depth of light. Step height technique was used in [8] to measure the phase change value; while in [9] the phase change was 
calculated using fringe counting. Interferometric technique in [10] ignored the wringing effect, while [11] ignored the roughness effect which do not represent the real case.

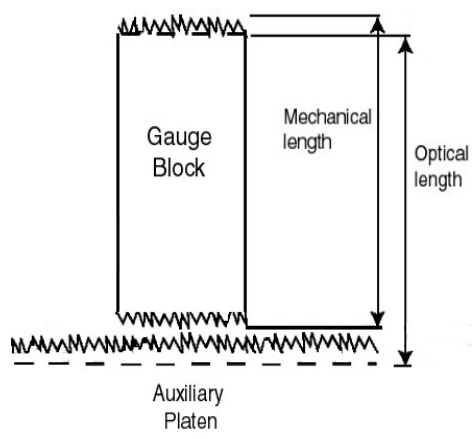

Fig. 1. Optical length includes the mechanical length, the wringing film, and the phase change at the gauge block and the auxiliary platen surfaces.

In order to reduce the errors resulted from the mechanical contact of the GB and the platen and the wringing film existing in the stack, another technique was suggested to measure the phase change correction using the integrating sphere [12].

In this paper, we used polarized light in measuring the optical constants of the steel GB by the principal angle of incidence method and used published data for the refractive index of the fused silica platen to make the required corrections. The proposed method is suitable to the contactless techniques such as the double ended interferometers that measure the GB length without wringing [13]. This enabled measuring the phase change correction without losing the advantage of the contactless method in the GB measurement. The standard uncertainty in measuring the phase change correction could be reduced from $\pm 5 \mathrm{~nm}$ [14] to $\pm 2 \mathrm{~nm}$ and the contribution of the other factors to the uncertainty was stated in Table $1 \mathrm{in}$ [14].

\section{Optical constants of the gauge block and the platen}

As a conducting material, the steel GB has a complex refractive index $\boldsymbol{n}$ defined in [15] as:

$$
\boldsymbol{n}=n_{g}\left(1-i \kappa_{g}\right)=n_{g}-i k_{g} ;
$$

where $\mathrm{n}_{\mathrm{g}}, \kappa_{g}$ and $\mathrm{kg}_{\mathrm{g}}$ refer to the refractive index, absorption index and extinction coefficients of the GB and $i$ is the imaginary unit $\left(i^{2}=-1\right)$. The optical constants of the steel GB were measured by the principal angle of incidence method. The optical system used is similar to that of a manual ellipsometer. However, it is not an ellipsometric method since we are not measuring the ellipsometric parameters $\psi$ and $\Delta$ (where $\tan \psi$ and $\Delta$ measure the change in the amplitude ratio and the change in phase upon reflection). The usual procedure is explained in [15].

In summary, He-Ne laser beam $\mathrm{L}(\lambda=632.8 \mathrm{~nm})$ crosses a polarizing prism $\mathrm{P}$ oriented with its transmission axis at $45^{\circ}$ and falls obliquely on the GB surface $G$ at angle of incidence $\phi$. The reflected beam passes successively through a quarterwave plate $C$ of retardance value $\pi / 2$ at $632.8 \mathrm{~nm}$ oriented with its fast axis at $0^{\circ}$, analyzing prism $\mathrm{A}$ and the detecting system $\mathrm{D}$ (photomultiplier, millivoltmeter and DC power supply), Figure 2.

Angles are measured in a counterclockwise (CCW) sense from the positive $\mathrm{x}$-direction by an observer receiving the radiation with respect to the transmission axes of $\mathrm{P}$ and $\mathrm{A}$ and the fast axis of $\mathrm{C}$. If linearly polarized light with azimuth $45^{\circ}$ falls obliquely on the surface of the gauge block at an angle of incidence $\phi$ less than the principal angle of incidence $\bar{\phi}$, it will be reflected in an elliptically polarized state with its principal axes not coincident with the $\mathrm{x}$ and $\mathrm{y}$ axes of the optical system. Increasing $\phi$ gradually, the polarization ellipse will rotate and its principal axes will approach the axes of the system. When $\phi=\bar{\phi}$, the ellipse assumes its standard form and its axes coincide with the system axes. Increasing $\phi$ above $\bar{\phi}$, the ellipse will continue rotating with its axes differing again from the system axes. At $\phi=\bar{\phi}$, the phase shift introduced between the $\mathrm{p}$ (parallel) and s (perpendicular) orthogonal components of the beam reflected from the GB is $90^{\circ}$. The phase plate $\mathrm{C}$ oriented with its fast axis at $0^{\circ}$ will convert this elliptical state into linear state that could be extinguished by rotating the analyzer A. The reading of the analyzer at extinction 
defines the principal azimuthal angle $\bar{\psi}$. Accoding to [15], the optical constants of the gauge block are defined by:

$$
\begin{gathered}
n_{g}=\sin \bar{\phi} \tan \bar{\phi} \cos 2 \bar{\psi} ; \\
k_{g}=n_{g} \tan 2 \bar{\psi}
\end{gathered}
$$

A major difficulty of this procedure is that in order to determine $\bar{\phi}$ precisely, a large number of trials is required (particularly around the value $\bar{\phi}$ ) by varying the angle $\phi$ and observing the orientation of the major axis each time.

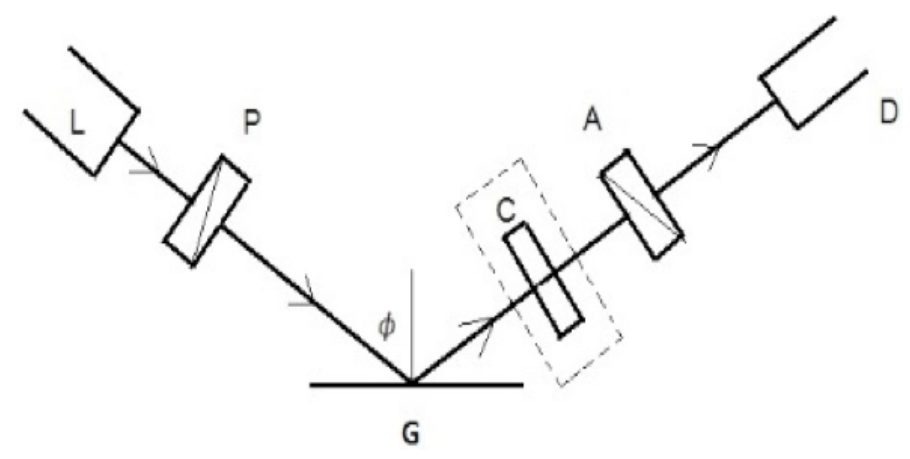

Fig. 2. The optical system used in the principal angle of incidence method.

Another serious difficulty is finding a plate of exact quarterwave retardance at the working wavelength $(632.8 \mathrm{~nm})$. Deviation of the retardance from the value $\pi / 2$ will introduce errors in the values of $\bar{\phi}$ and $\bar{\psi}$ and consequently in the values of the optical constants. The effect of retardance deviation from the value $90^{\circ}$ on the optical constants of a metal was discussed in [16] and considered as a main factor for the wide differences in the published results.

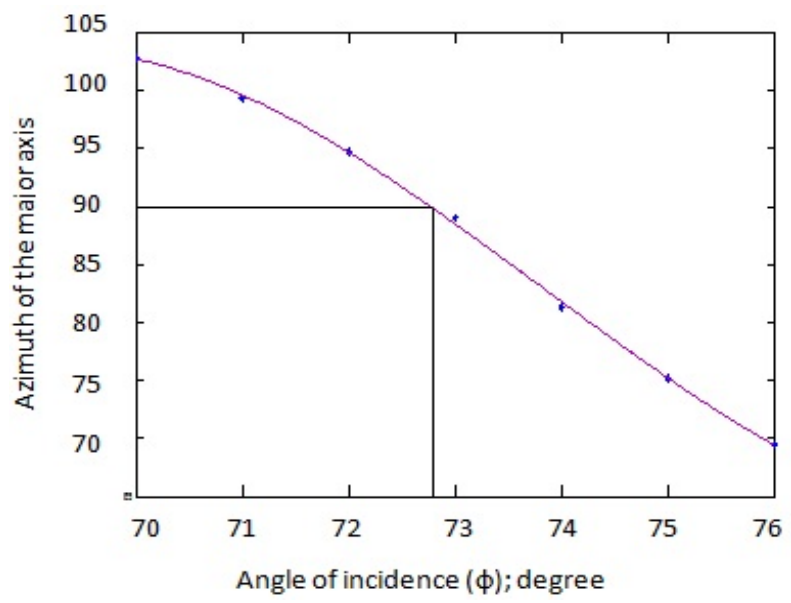

Fig. 3. The relation between the angle of incidence $\phi$ and the azimuth of the polarization ellipse.

To overcome these difficulties, we followed a two-step procedure [17] by first removing the phase plate from the system and performing measurements starting from $70^{\circ}$ in steps of $1^{\circ}$ (for conductors $\bar{\phi}$ usually exceeds $70^{\circ}$ ). The orientation of the major axis was recorded each time. Measurements continued until the polarization ellipse crossed the state of standard form. The relation between the angle of incidence and the azimuth of the polarization ellipse (determined at the maximum intensity reached by rotating the analyzer) is shown in Figure.3.

It was concluded that

$$
\bar{\phi}=72.75^{\circ}
$$

Next, the phase plate was introduced in the system with $\phi$ set at the principal angle value and A is rotated for extinction. The principal azimuthal angle was found as 


$$
\bar{\psi}=23.83^{\circ}
$$

Note that a reasonable deviation of the plate retardance from the value $\pi / 2$ will have a negligible effect on the value of $\bar{\psi}$. This deviation will cause a small ellipticity in the beam incident on A. Extinction by A will not then be complete, but it is still possible to define $\bar{\psi}$ precisely as a very thin elliptical state behaves practically as a plane polarized state. It follows from Eqs. $2(a, b)$ that

$$
\begin{aligned}
& \mathrm{n}_{\mathrm{g}}=2.0715 \\
& \mathrm{k}_{\mathrm{g}}=2.2734
\end{aligned}
$$

The refractive index of the fused silica platen $\mathrm{n}_{\mathrm{p}}=1.4570$ at $632.8 \mathrm{~nm}$ [18] and its extinction coefficient $\mathrm{k}_{\mathrm{p}}$ $=0$ in the visible spectrum. The major contributors to the accuracy in determining the optical constants of the steel GB are the precision of adjusting the scales of the polarizing prisms and errors in determining the angles $\bar{\phi}$ and $\bar{\psi}$.

\section{Calculation of Phase Change Correction}

The beams reflected from the GB and the platten encounter phase change $\delta$ defined by $[10,19]$

$$
\tan \delta=\frac{2 n_{1} k_{2}}{n_{1}^{2}-n_{2}^{2}-k_{2}^{2}}
$$

where $n_{1}$ is the refractive index of the incident medium (air), $n_{2}$ is the refractive index of the reflective medium (GB or platen), and $k_{2}$ the extinction coefficient of the reflective medium (GB or platen).

From the calculated values of optical constants, the measured phase change for steel is $152^{\circ}$, while for the fused silica is $180^{\circ}$. The measured constants of our materials are comparable to values in [20-22].

The phase correction of the fused quartz platen is then zero, and the correction value will result from the GB only. This is reasonable since for the fused quartz platen, $\mathrm{k}_{2}$ in Eq.3 is zero at all wavelengths in the visible spectrum. The corresponding length $(\Delta L)$ to the measured phase change on reflection is found to be $24.6 \mathrm{~nm}$ from the equation

$$
\Delta \delta=\frac{2 \pi}{\lambda}(2 \Delta L)
$$

The uncertainty in measuring the phase change angle is summarized in table 1 according to [23].

The combined uncertainty is calculated as $u_{c}(\delta)= \pm 2^{\circ}$, and its contribution to the uncertainty in gauge block length measurement is $\pm 2 \mathrm{~nm}$. This value is compared with the phase change correction value for the same GB and auxiliary platen measured by the stack method using Kösters interference comparator $(20 \mathrm{~nm} \pm 5 \mathrm{~nm})$.

\begin{tabular}{|c|c|c|c|}
\hline $\begin{array}{c}\text { TABLE 1. Uncertainty in measuring the phase change correction } \\
\text { of uncertainty }\end{array}$ & $x_{i}$ & $\begin{array}{c}\text { Standard } \\
\text { uncertainty } \\
u\left(x_{i}\right)\end{array}$ & $u\left(y_{i}\right)$ \\
\hline Polarizer Reading & $\pm 0.016^{\circ}$ & $0.009^{\circ}$ & $\pm 0.009^{\circ}$ \\
\hline Analyzer Reading & $\pm 0.048^{\circ}$ & $0.027^{\circ}$ & $\pm 0.027^{\circ}$ \\
\hline Refraction Coeff. & & & $\pm 1.66^{\circ}$ \\
$n(\bar{\varphi}, \bar{\psi})$ & 2.0715 & \pm 0.114 & $( \pm 0.07)$ \\
$\bar{\varphi}$ & $\left( \pm 0.016^{\circ}\right)$ & $\left(0.009^{\circ}\right)$ & $( \pm 0.09)$ \\
$\bar{\psi}$ & $\left( \pm 0.033^{\circ}\right)$ & $\left(0.02^{\circ}\right)$ & $\pm 0.513^{\circ}$ \\
\hline Extinction Coeff. & & & $( \pm 0.08)$ \\
$K(\bar{\varphi}, \bar{\psi})$ & 2.273 & \pm 0.19 & $( \pm 0.17)$ \\
$\bar{\varphi}$ & $\left( \pm 0.016^{\circ}\right)$ & $\left(0.009^{\circ}\right)$ & \\
$\bar{\psi}$ & $\left( \pm 0.033^{\circ}\right)$ & $\left(0.02^{\circ}\right)$ & \\
\hline
\end{tabular}




\section{Conclusion}

The values of the phase change on reflection of a steel GB and fused silica optical flat are evaluated. The study depends on measurements of the optical constants of the concerned materials using polarized light. We introduced a modification to the principal angle method to increase the accuracy of measuring the optical constants of the steel GB to avoid the usually expected deviation of the quarterwave plate from exact $\lambda / 4$ value. This resulted in a serious reduction in the uncertainty in the measured values of $n$ and $k$ for the steel GB and consequently in the uncertainty in the measured phase correction. The uncertainty in measurement is evaluated and the results are compared with those obtained by the stack method. The contribution of the phase change correction parameter to the expanded uncertainty in gauge block calibration is reduced to $\pm 2 \mathrm{~nm}$. This method of measuring the phase change correction does not require any mechanical contact to the gauge block surface and is convenient to the contactless measurement of the gauge block length using the double ended interferometer. 